\title{
Perturbed Dual Semigroups and Delay Equations
}

\author{
Odo Diekmann \\ Centre for Mathematics and Computer Science \\ P.O. Box 4079, 1009 AB Amsterdam, The Netherlands \\ and \\ Institute of Theoretical Biology \\ University of Leiden \\ Groenhovenstraat 5 \\ 2311 BT Leiden, The Netherlands
}

\begin{abstract}
The theory of dual semigroups on non-reflexive Banach spaces can be used to define a natural generalization of the notion of a bounded perturbation of the generator and a new version of the variation - of - constants formula. This approach was developed in joint work with Ph. Clément, M. Gyllenberg, H.J.A.M. Heijmans and H.R. Thieme, motivated by some applications to physiologically structured population growth models. In this paper it is shown that delay differential equations fit very well into exactly the same functional analytic framework.
\end{abstract}

1. Introduction.

The variation - of - constants formula is a very convenient starting point for the derivation of many results in the local stability and bifurcation theory of ordinary and partial differential equations. This statement is equally valid for retarded functional differential equations, but here the variation - of constants formula shows a peculiar feature. Indeed, in the book of HALE [6] we find that the formula involves the so-called fundamental matrix solution $X$ which is, by definition, the solution corresponding to the special discontinuous initial condition $X(t)=0$ for $t<0$ and $X(0)=I$ (the identity matrix) and which, therefore, does not "live" in the state space $C$. As a consequence one has to interpret the convolution integral which figures in the variation - of - constants formula as a family (parametrized by the independent variable of the functions in $C$ ) of integrals in Euclidean space. Thus the formula becomes symbolic rather than functional analytic (it does not fit into the standard semigroup framework).

The difficulty resolves to some extent if one embeds $C$ into the product space $M_{p}=\mathbb{R}^{n} \times L_{p}$ (see, for instance, KAPPEL and SCHAPPACHER [8] ). The fundamental matrix solution is well-defined in the $M_{p}$ context and so is the convolution integral which maps $C$ into $C$ (here we identify $C$ with its embedding into $M_{p}$ ). Actually the fact that the variation - of - constants formula makes sense in $M_{p}$ is the main motivation for introducing this space (see DELFOUR and MANITIUS [2] and the references given there).

Another class of equations for which the variation - of - constants formula is not directly available comes up in population biology. If one considers age-dependent population growth models with possibly nonlinear birth terms the stability and bifurcation theory is troubled by annoying technical difficulties, as one may notice by reading the work of GURTIN and MACCAMY [5], PRÜB [11] and WEBB [13]. There is, actually, much more similarity with the case of delay equations than appears from these works. The solution which has the (Dirac) measure concentrated at age zero as initial condition is well-defined and plays exactly the same role as the fundamental solution for delay equations. Again the trouble is that this specially important initial condition does not exist in the conventional state space $L_{1}\left(\mathbb{R}_{+}\right)$. An identical picture emerges : even though the integrand in the convolution term of the variation - of - constants formula does not live in the state space $X$ but in some larger space the convolution integral itself defines an element of $X$.

This observation triggered Ph. Clément, M. Gyllenberg, H.J.A.M. Heijmans, H.R. Thieme and myself to investigate the phenomenon from a functional analytic point of view. Motivated by the duality between the Kolmogorov forward and backward formulations of linear age dependent population dynamics we studied the existing theory of dual semigroups on (non-reflexive) Banach spaces. We found that within this framework one can give a very natural generalization of the notion of a bounded perturbation of the generator and that this leads to a new version of the variation - of - constants formula. In sections 2 and 3 I will give a summary of the preprint [1]. In section 4 I show that delay equations fit into the framework. It will appear that one can "construct" the space $M_{\infty}$ starting from $C$ and the very simple semigroup generated by $\dot{x}=0$ considered as a delay equation, that it is 
natural to embed $C$ into $M_{\infty}$, and that one has to interpret the convolution integral in a weak * sense. Thus we arrive at a functional analytic underpinning of the standard variation - of - constants formula for delay equations.

There is yet another way in which the basic difficulty manifests itself in both delay equations and age dependent population growth equations. The domain of the semigroup generator involves much more than just a smoothness condition. In fact for delay equations all information about the particular equation is contained in the domain, whereas the action of the generator does not depend on the equation at all. This aspect of the problem will be explained as well. The domain of the weak $*$ generator on $M_{\infty}$ is independent of the particular equation and involves a smoothness condition only. When taking the restriction to $C$ we have to restrict the range of the generator and this causes a shift of information from action to domain.

The problems motivating [1] are identical to some of the problems motivating recent work by DESCH and SCHAPPACHER (see [3] and the references given there), but the solution we propose is different (although there are some common characteristics).

\section{Dual semigroups.}

Let $\{T(t)\}$ be a strongly continuous semigroup of bounded linear operators on a Banach space $X$ and let $A$ denote its generator. The adjoint operators $T^{\star}(t)$ form a semigroup on the dual space $X^{\star}$. The semigroup $\left\{T^{*}(t)\right\}$ is weak $\star$ continuous. But if we equip $X^{\star}$ with the usual norm topology $\left\{T^{*}(t)\right\}$ need not be strongly continuous (unless $X$ is reflexive). The operator $A^{*}$, the adjoint of $A$, is the weak * generator of $\left\{T^{*}(t)\right\}$ but need not be densely defined.

In their classic treatise HILle and PHILlIPs [7] showed that the dialogue of a space and a semigroup demands a duality theory which is made to measure. We need a special star, called sun and represented by the symbol $\odot$. Let

$$
X^{\odot}=\left\{x^{*} \in X^{*} \mid \lim _{t \downarrow 0}\left\|T^{*}(t) x^{*}-x^{\star}\right\|=0\right\} .
$$

Then $X^{\odot}$ is the maximal invariant subspace on which $\left\{T^{*}(t)\right\}$ is strongly continuous, $X^{\odot}$ is norm closed and $X^{\odot}=\widehat{D\left(A^{*}\right)}$. Let $\left\{T^{\odot}(t)\right\}$ denote the strongly continuous semigroup on $X^{\odot}$ which is obtained by restriction of $\left\{T^{*}(t)\right\}$ and let $A^{\odot}$ denote its generator. Then $A^{\odot}$ is the part of $A^{*}$ in $X^{\odot}$, i.e. the largest restriction of $A^{*}$ with both domain and range in $A^{\odot}$.

Repeating the same procedure we obtain a weak $\star$ continuous semigroup $\left\{T^{\odot *}(t)\right\}$ on $X^{\odot *}$, the dual space of $X^{\odot}$, with weak $\star$ generator $A^{\odot \star}$. Let

$$
X^{\odot \odot}=\left\{x^{\odot *} \in X^{\odot *} \mid \lim _{t \downarrow 0}\left\|T^{\odot *}(t) x^{\odot *}-x^{\odot *}\right\|=0\right\} .
$$

A straightforward reinterpretation of the duality pairing between elements of $X$ and $X^{\odot}$ yields a natural embedding of $X$ into $X^{\odot *}$ and henceforth we identify $X$ and this embedding into $X^{\odot *}$. Then $X$ becomes a subspace of $X^{\odot \odot}$.

Definition : $X$ is called $\odot$-reflexive with respect to $A$ iff $X=X^{\odot \odot}$.

A slightly more careful formulation is obtained if we first equip $X$ with the equivalent norm

$$
\|x\|^{\prime}=\sup \left\{\left|<x, x^{\odot}\right\rangle \mid: x^{\odot} \in X^{\odot},\left\|x x^{\odot}\right\| \leqslant 1\right\} .
$$
But if $\{T(t)\}$ is a contraction semigroup, which it is in our application to delay equations, the two
norms are actually the same.

It is known that $X$ is $\odot$-reflexive with respect to $A$ iff $(\lambda \mathrm{I}-A)^{-1}$ is $X^{\odot}$-weakly compact. Moreover, $X$ is $\odot$-reflexive with respect to $A$ iff $X^{\odot}$ is $\odot$-reflexive with respect to $A^{\odot}$.

3. Perturbation theory.

Let $\left\{T_{0}(t)\right\}$ be a strongly continuous semigroup on $X$ generated by $A_{0}$ and assume that $X$ is $\odot$ reflexive with respect to $A_{0}$. We want to perturb the generator $A_{0}$ by a linear operator $B$, where $B$ is bounded as an operator from $X$ into $X^{\odot *}$. To this end we consider the variation - of - constants
equation

$$
T(t) x=T_{0}(t) x+\int_{0}^{t} T_{0}^{\odot *}(t-\tau) B T(\tau) x d \tau
$$


Here the integral has to be understood in the weak * sense, i.e.

$$
<\int_{0}^{t} T_{0}^{\odot *}(t-\tau) B T(\tau) x d \tau, x^{\odot}>:=\int_{0}^{t}<B T(\tau) x, T_{0}^{\odot}(t-\tau) x^{\odot}>d \tau
$$

for arbitrary $x^{\odot} \in X^{\odot}$. So in principle the integral takes values in $X^{\odot *}$ but one can show that in fact it takes values in the closed subspace $X^{\odot \odot}=X$. Within this setting the standard contraction arguments apply and one infers that (3.1) admits a unique solution $\{T(t)\}$. By duality and restriction we obtain semigroups $\left\{T^{*}(t)\right\},\left\{T^{\odot}(t)\right\}$ and $\left\{T^{\odot *}(t)\right\}$ on, respectively $X^{*}, X^{\odot}$ and $X^{\odot *}$, since it can be shown that the spaces of strong continuity do not depend on $B$ ! Similarly the domains of the weak * generators on the "big" spaces $X^{*}$ and $X^{\odot *}$ are independent of $B$.

THEOREM. The operator $A x=A_{0}^{\odot *} x+B x$ with $D(A)=\left\{x \in D\left(A_{0}^{\odot *}\right): A_{0}^{\odot *} x+B x \in X\right\}$ is the generator of a strongly continuous semigroup $\{T(t)\}$ on $X$ and the variation - of - constants formula (3.1) holds.

Next assume that the operator $B$ has finite dimensional range. So let there be given $r_{1}^{\odot *}, \ldots, r_{n}^{\odot *} \in X^{\odot *}$ and $r_{1}^{*}, \ldots, r_{n}^{*} \in X^{*}$ such that

$$
B x=\sum_{i=1}^{n}<r_{i}^{*}, x>r_{i}^{\odot *} .
$$

Let $Q$ denote a $n \times n$-matrix-valued function with entries

$$
q_{i j}(t)=\left\langle r_{i}^{*}, \int_{0}^{t} T_{0}^{\odot *}(\tau) r_{j}^{\odot *} d \tau\right\rangle
$$

A simple estimate shows that $Q$ is Lipschitz continuous. As a consequence we have a representation of the form

$$
Q(t)=\int_{0}^{t} K(\tau) d \tau
$$

where the entries $k_{i j}$ of $K$ belong to $L_{\infty}$. By a roundabout way we thus gave a meaning to $<r_{i}^{*}, T_{0}^{\odot *}(t) r_{j}^{\odot *}>$, though as a function of $t$ and not pointwise in $t$ !

Define the $n$-vector $y(t)$ by

$$
y_{i}(t)=\left\langle r_{i}^{*}, T(t) x\right\rangle
$$

where $T(t) x$ is the solution of (3.1). Equation (3.1) and a little technical calculation (to avoid undefined expressions) imply that $y$ satisfies the renewal equation

$$
y=h+K \star y
$$

where the $n$-vector valued forcing function $h$ is given by

$$
h_{i}(t)=\left\langle r_{i}^{*}, T_{0}(t) x\right\rangle
$$

and $K \star y$ denotes the convolution product of $K$ and $y$. Conversely, given any solution $y$ of (3.6) with $h$ of the form (3.7) we can recover $T(t) x$ from

$$
T(t) x=T_{0}(t) x+\sum_{j=10}^{n} \int_{0}^{t} T_{0}^{\odot *}(t-\tau) r_{j}^{\odot *} y_{j}(\tau) d \tau
$$

It appears that solving (3.1) is reduced to solving (3.6)

Let $B^{*}: X^{\odot} \rightarrow X^{*}$ denote the (restriction of the) adjoint of $B$. The semigroup $\left\{T^{\odot}(t)\right\}$ satisfies the "adjoint" variation - of - constants equation

$$
T^{\odot}(t) x^{\odot}=T_{0}^{\odot}(t) x^{\odot}+\int_{0}^{t} T_{0}^{*}(t-\varangle) B^{*} T^{\odot}(\tau) x^{\odot} d \tau
$$

So if

$$
B^{*} x^{\odot}=\sum_{i=1}^{n} r_{i}^{*}<x^{\odot}, r_{i}^{\odot *}>
$$


then the $n$-vector valued function $z$ defined by

$$
z_{i}(t)=\left\langle T^{\odot}(t) x^{\odot}, r_{i}^{\odot *}\right\rangle
$$

satisfies the "adjoint" renewal equation

$$
z=g+K^{T} \star z
$$

where $K^{T}$ denotes the transpose of $K$ (if the entries are complex we have to take complex conjugates as well) and the forcing function $g$ is defined by

$$
g_{i}(t)=\left\langle T_{0}^{\odot}(t) x^{\odot}, r_{i}^{\odot *}\right\rangle
$$

Again one can recover the full semigroup from a knowledge of $z$ only.

The symmetry of the present framework can be expressed in a diagram :

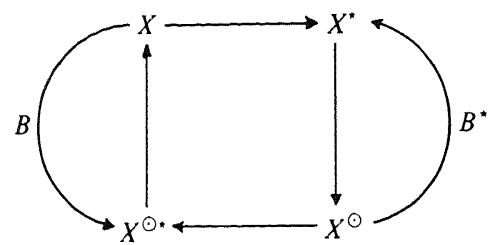

where horizontal arrows indicate going over to the dual space (taking adjoints) and vertical arrows indicate taking restrictions to the maximal space of strong continuity. When $X$ is not $\odot$-reflexive with respect to $A_{0}$ this symmetry is disturbed. Nevertheless similar results hold. A canonical embedding of $X^{\odot \odot}$ into $X^{* *}$ seems to play a leading part, but it is not yet precisely clear how the most elegant and efficient argumentation proceeds, so we refrain from further discussion here.

4. Retarded functional differential equations.

Let $\zeta$ be a given $n \times n$-real-matrix-valued function of bounded variation such that $\zeta(\theta)=0$ for $\theta \leqslant 0$ and $\zeta(\theta)=\zeta(1)$ for $\theta \geqslant 1$. Here and in the following we assume that all bounded variation functions are normalized such that they are right continuous on $(0,1)$, zero on $(-\infty, 0]$ and constant on $[1, \infty)$. We consider the linear retarded functional differential equation

$$
\dot{x}(t)=\int_{0}^{1} d \zeta(\tau) x(t-\tau)
$$

with initial condition

$$
x(\theta)=\phi(\theta),-1 \leqslant \theta \leqslant 0,
$$

where $\phi \in X=C\left[[-1,0] ; \mathbb{R}^{n}\right]$. The semigroup $\left\{T_{\zeta}(t)\right\}$ on $X$ is defined by

$$
\left(T_{\zeta}(t) \phi\right)(\theta)=x_{t}(\theta ; \phi)
$$

where $x(t ; \phi)$ denotes the unique solution of $(4.1)-(4.2)$ and, as usual, $x_{t}(\theta, \phi)=x(t+\theta ; \phi)$. The action of $T_{\zeta}(t)$ is built from two constituents : translation and a rule for extension. An easy prototype is obtained by making the rule for extension as simple as possible, that is by taking $\zeta \equiv 0$. So let us take as the unperturbed problem the equation $\dot{x}=0$ considered as a delay equation. The semi-
group

$$
\left(T_{0}(t) \phi\right)(\theta)= \begin{cases}\phi(\theta+t) & , \quad \theta+t \leqslant 0 \\ \phi(0) & , \quad \theta+t \geqslant 0\end{cases}
$$

on $X$ is generated by

$$
A_{0} \phi=\phi^{\prime}, \quad D\left(A_{0}\right)=\left\{\phi \in C^{1}: \phi^{\prime}(0)=0\right\}
$$

Let $X^{*}$ be represented by $N B V\left([0,1] ; \mathbb{R}^{n}\right)$ with the pairing

$$
\langle f, \phi\rangle=\int_{0}^{1} d f(\tau) \phi(-\tau)
$$


Then

$$
\left(T_{0}^{*}(t) f\right)(\sigma)=f(\sigma+t), \sigma>0,
$$

and

$$
A_{0}^{*} f=f^{\prime}, D\left(A_{0}^{*}\right)=\left\{f: f \in A C, f^{\prime} \in N B V\right\},
$$

where $A C$ abbreviates "absolutely continuous". Hence

$$
X^{\odot}=\overline{D\left(A_{0}^{*}\right)}=A C=\mathbb{R}^{n} \oplus A C_{0}=\left\{f: f(t)=c+\int_{0}^{t} g(\tau) d \tau, g \in L_{1}, \text { supp } g \subset[0,1]\right\}(4
$$

It is sometimes convenient to work with the couple $(c, g)$ to represent $f$. This amounts to representing $X^{\odot}$ by $\mathbb{R}^{n} \times L_{1}$, where $L_{1}=\left\{g \in L_{1}\left(\mathbb{R}_{+}\right): \operatorname{supp} g \subset[0,1]\right\}$, with norm $\|(c, g)\|=|c|+\|g\|_{L_{1}}$. In these coordinates we have

$$
\begin{aligned}
& T_{0}^{\odot}(t)(c, g)=\left[c+\int_{0}^{t} g(\tau) d \tau, g(t+\cdot)\right] \\
& A_{0}^{\odot}(c, g)=\left(g(0), g^{\prime}\right), D\left(A_{0}^{\odot}\right)=\{(c, g): g \in A C\}
\end{aligned}
$$

Next we take the representation $X^{\odot *}=M_{\infty}=\mathbb{R}^{n} \times L_{\infty}\left([-1,0] ; \mathbb{R}^{n}\right)$ with norm $\|(\alpha, \phi)\|=\sup \left\{|\alpha|,\|\phi\|_{L_{\infty}}\right\}$ and pairing

$$
<(c, g),(\alpha, \phi)>=c \alpha+\int_{0}^{1} g(\tau) \phi(-\tau) d \tau .
$$

It follows that $T_{0}^{\odot *}(t)$ is the shift of the $\alpha$-extended $\phi$ :

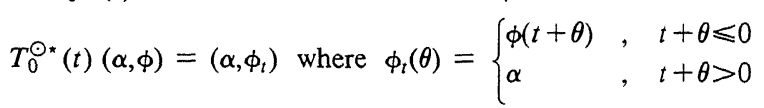

and

$$
A_{0}^{\odot *}(\alpha, \phi)=\left(0, \phi^{\prime}\right), D\left(A_{0}^{\odot *}\right)=\{(\alpha, \phi): \phi \in \operatorname{Lip}(\alpha)\}
$$

where $\operatorname{Lip}(\alpha)$ denotes the class of elements of $L_{\infty}$ which contain a Lipschitz continuous function which assumes the value $\alpha$ at $\theta=0$. Finally,

$$
X^{\odot \odot}=\overline{D\left(A_{0}^{\odot *)}\right.}=\{(\alpha, \phi): \phi \in C(\alpha)\}
$$

where $C(\alpha)$ denotes the class of elements of $L_{\infty}$ which contain a continuous function which assumes the value $\alpha$ at $\theta=0$. The embedding of $X$ into $X^{\odot *}$ is described by $j \phi=(\phi(0), \tilde{\phi})$, where $\tilde{\phi}$ denotes the $L_{\infty}$ equivalence class to which $\phi$ belongs. Clearly $j(X)=X^{\odot \odot}$. We conclude that $X$ is $\odot$ reflexive with respect to $A_{0}$, a fact which can also be deduced from the compactness of $\left(\lambda I-A_{0}\right)^{-1}$.

So far we have used the semigroup $\left\{T_{0}(t)\right\}$ to construct a dual space $X^{\odot *}=M_{\infty}$ in which $X=C$ lies embedded. Next we are going to perturb the generator by changing the rule for the extension of the function. The space $C$ is too small to describe this perturbation but the space $M_{\infty}$ is large enough.

Define $B: C \rightarrow M_{\infty}$ by

$$
B \phi=(<\zeta, \phi>, 0)
$$

Clearly the results of section 3 apply. In particular the theorem implies that for given $\zeta$ we have a semigroup $\{T(t)\}$ generated by the operator $A$ with domain

$$
D(A)=\left\{\phi \in \operatorname{Lip}: \phi^{\prime} \in C(<\zeta, \phi>)\right\}=\left\{\phi \in C^{1}: \phi^{\prime}(0)=\langle\zeta, \phi>\}\right.
$$

and action $A \phi=\phi^{\prime}$. It is well-known that the same operator $A$ generates the semigroup $\left\{T_{\zeta}(t)\right\}$ introduced at the beginning of section 4 and consequently the semigroups are really the same. We prefer to give another more direct proof of this fact which does not require any knowledge about the generator of $\left\{T_{\zeta}(t)\right\}$.

The element $r_{i}^{*}$ is the $i$-th row of $\zeta$ and $r_{i}^{\odot *}=\left(e_{i}, 0\right)$, where $e_{i}$ denotes the $i$-th unit column vector in $\mathbb{R}^{n}$. It is convenient to combine these into matrices $r^{*}=\zeta$ and $r^{\odot *}=(I, 0)$. Then

$$
T_{0}^{\odot *}(t) r^{\odot *}=(I, H(t+\cdot) I),
$$


where $H$ denotes the Heaviside function. Substituting this into (3.8) we find

$$
(T(t) \phi)(\theta)=\left(T_{0}(t) \phi\right)(\theta)+\int_{0}^{\max \{0, t+\theta\}} y(\tau) d \tau
$$

So if we define for $t \geqslant 0$

$$
x(t ; \phi)=(T(t) \phi)(0)
$$

then $x(t ; \phi)=\phi(0)+\int_{0}^{t} y(\tau) d \tau$ from which it follows that $y(t)=\dot{x}(t ; \phi)$ and, moreover, (4.16) then implies that for $t+\theta \geqslant 0$

$$
(T(t) \phi)(\theta)=\phi(0)+\int_{0}^{t+\theta} y(\tau) d \tau=x(t+\theta ; \phi) .
$$

It remains to calculate the kernel $K$ and the forcing function $h$ in the renewal equation (3.6) for $y$. From (4.15) we deduce that

$$
\int_{0}^{t} T_{0}^{\odot *}(\tau) r^{\odot *} d \tau=(t+\cdot)_{+} I
$$

where $(\theta)_{+}:=\max \{0, \theta\}$. Hence

$$
M(t)=<\zeta,(t+\cdot)_{+} I>=\int_{0}^{t} d \zeta(\tau)(t-\tau)=\int_{0}^{t} \zeta(\sigma) d \sigma
$$

from which it follows that

$$
K(t)=\zeta(t) \text {. }
$$

Finally

$$
h(t)=\left\langle r^{*}, T_{0}(t) \phi>=\int_{t}^{1} d \zeta(\tau) \phi(t-\tau)+\zeta(t) \phi(0) .\right.
$$

Now observe that one may start from (4.1) - (4.2) and manipulate as follows :

$$
\begin{aligned}
\dot{x}(t) & =\int_{0}^{t} d \zeta(\tau) x(t-\tau)+\int_{t}^{1} d \zeta(\tau) \phi(t-\tau) \\
& =\int_{0}^{t} d \zeta(\tau) \dot{x}(t-\tau) d \tau+\zeta(t) \phi(0)+\int_{t}^{1} d \zeta(\tau) \phi(t-\tau)
\end{aligned}
$$

to obtain exactly the same renewal equation for $\dot{x}=y$. Once more we attain to the conclusion that our semigroup is the solution semigroup corresponding to the problem (4.1) - (4.2).

In conclusion of this section I show how the present approach yields rather directly a suitable interpretation of the dual semigroup. The action of $r^{\odot *}$ corresponds to taking the limit from above in zero. So (3.13) implies that for $t>0$

$$
g(t)=\left(T_{0}^{\odot}(t) f\right)(0+)=f(t+)=f(t)
$$

or, in other words, the forcing function in the renewal equation and the state in our dynamical framework are one and the same thing! According to (3.9) and (3.11) we have

$$
\left(T^{\odot}(t) f\right)(\sigma)=f(t+\sigma)+\int_{0}^{t} \zeta^{T}(t-\tau+\sigma) z(\tau) d \tau .
$$

On the other hand we may start from the renewal equation $z=f+\zeta^{T} \star z$ and define a semigroup $\{S(t)\}$ by requiring that

$$
z_{t}=S(t) f+\zeta^{T} \star z_{t}
$$

that is, $S(t) f$ is the new forcing function in the renewal equation for the translated function $z_{t}$. A straightforward computation shows that $T^{\odot}(t)=S(t)$. Note, finally, that the renewal equation $z=f+\zeta^{T} \star z$ is obtained from the delay equation $\dot{z}(t)=\left\langle\zeta^{T}, z_{t}\right\rangle$ by integrating the renewal 
equation for $\dot{z}$.

Further comments on duality for delay equations may be found in $[2,4]$ and the references given there.

5. Concluding remarks.

The present note concentrates on linear equations. It should be clear, however, that one can deal in a similar spirit with Lipschitz continuous operators from $X$ into $X^{\odot *}$ and that results on linearized stability, the center manifold etc. can be proved in the standard manner. Nonlinear retarded functional differential equations are semilinear in the sense of section 3 !

A minor but curious point is that the duality framework shows so easily that even differentialdifference equations such as $\dot{x}(t)=x\left(t-\frac{1}{2}\right)$ admit well-defined solutions when the initial function is given as an element of $M_{\infty}$ only.

Equations with infinite delay don't require the full machinery of the non- $\odot$-reflexive case simply because $X$ is again invariant under the perturbed semigroup. As yet I have made no attempt to eleborate the details. Recently NaITo [10] and MURAKami [9] have employed the second dual of $X$ and the weak * integral to define the variation - of - constants formula for equations with infinite delay.

My unfamiliarity with neutral equations keeps me from investigating whether or not the present framework has anything to offer for those and I welcome any aficionado who is willing to do so.

In the recent paper [12] and in work in progress Verduyn Lunel" studies the renewal equations (3.6) and (3.12) using the Laplace transform and complex function theory as his main tools. As corollaries he obtains strong results concerning the existence or non-existence of solutions which vanish identically after a given finite time and concerning the characterization of $\overline{\mathscr{T}}$, the closure of the linear span of all eigenfunctions.

\section{REFERENCES}

[1] Ph. Clément, O. Diekmann, M. Gyllenberg, H.J.A.M. Heimmans and H.R. Thieme, Perturbation theory for dual semigroups. I. The $\odot$-reflexive case. Preprint.

[2] M.C. Delfour and A. Manitius, The structural operator $F$ and its role in the theory of retarded systems. J. Math. Anal. Appl. I. 73 (1980) 466-490, II. 74, (1980) 359-381.

[3] W. DESCH and W. SCHAPPACHER, Spectral properties of finite-dimensional perturbed linear semigroups. J. Diff. Equ. 59 (1985) 80-102.

[4] O. Diekmann, A duality principle for delay equations, Proceedings of Equadiff 5, M. Gregus (ed.), Teubner 47 (1982) 84-86.

[5] M.E. GurTin and R.C. MACCAMY, Nonlinear age-dependent population dynamics, Arch. Rat. Mech. Anal. 54 (1974) 281-300.

[6] J.K. Hale, Theory of Functional Differential Equations, Springer, 1977.

[7] E. Hille and R.S. PHILlips, Functional Analysis and Semi-groups, Amer. Math. Soc., Providence, 1957.

[8] F. KAPPEL and W. SCHAPPACHER, Non-linear functional differential equations and abstract integral equations, Proc. Roy. Soc. Edinburgh, 84 A (1979) 71-91.

[9] S. MURAKAMI, Linear periodic functional differential equations with infinite delay, to appear.

[10] T. NAITO, A modified form of the variation - of - constants formula for equations with infinite delay, Tôhoku Math. J. 36 (1984) 33-40.

[11] J. PRUß, Stability analysis for equilibria in age-specific population dynamics, Nonl. Anal., Th., Meth. Appl. 7 (1983) 1291-1313.

[12] S.M. VERDUYN LUNEL, A sharp version of Henry's theorem on small solutions, J. Diff. Equ. 62 (1986) 266-274.

[13] G.F. WeBb, Theory of Nonlinear Age-Dependent Population Dynamics, Marcel Dekker, 1985. 